\title{
DEGRADATION OF VDMOSFET BY HEAVY ION IRRADIATIONS
}

\author{
C. SALAME*, F. PELANCHON and P. MIALHE \\ Centre d'Etudes Fondamentales, Université de Perpignan, 52- av de \\ Villeneuve-66860, Perpignan Cedex., France
}

(Received 28 December 1999; In final form 28 January 2000)

\begin{abstract}
This article focuses on the effect of the heavy ions irradiations on the electrical characteristics of VDMOSFET (Vertical Diffusion Metal Oxide Semiconductor Field Effect Transistor) devices. A summary of the total dose effects and the single event effects is covered to evaluate the experimental observations. Device degradations due to the hot carriers junction avalanche are studied by a physical parameters extraction method, leading to an understanding of the degradation processes. Results show that a protective resistor load can reduce the degradation effect in the device.
\end{abstract}

Keywords: Hot-holes; transistor degradation; fast surface states; ionization radiation

\section{INTRODUCTION}

Microcircuits in space and nuclear environment interact with various particles, including galactic cosmic rays, radiation belt protons, electrons, neutrons and ions, all of which can affect the normal operations of the devices. Functional disturbances, arising from interactions with single high-energy particles, are often called "single-event effects" (SEE's). Microcircuit functions can also be compromised by other radiation effects than SEE, such as the displacement damage and the dose effects. Displacement damage is mainly caused by interaction of high energy incident particles with the material creating vacancies and interstitial, which are the direct results of displaced

*Corresponding author. e-mail: salame@ieee.org 
atoms in the crystal. Dose effects result from any radiation interaction and are generally interpreted by the accumulation of induced trapped charges and interface states.

In order to ensure that space system electronics will complete their mission without a radiation-induced failure, a program test is required. This program will usually involve radiation effects characterization of sensitive electronic devices as well as the development of the fragment hardness assurance approach for critical parts. Thus, it is necessary to perform a radiation testing to understand the failure mechanisms and to characterize the radiation response of specific devices.

Dose effects are discussed to point out the consequences of the major basic degradation processes on the fundamental parameters of the devices. We evaluate the effect of the drain avalanche hot hole injection on the electrical parameters of the VDMOSFET when a heavy ion passes through the devices biased in the off state (blocking a high-drain-source voltage).

In order to explain our experimental results, a synthetic presentation, in part 2, of the irradiation induced effects allows us to analyze the process involved in the device degradation. These results show that a protective resistor load $\left(R_{L}\right)$ can reduce the degradation effect in the device resulting from the interaction of a single high-energy heavy ion (HI) passing through the device. A summary of the single event effects is done in part 2 to clarify the role of this protective resistor. An original method presented in part 3 is developed which considers the structural and electrical parameters of the drain-body junction in order to follow up and discuss the observed degradation described in part 4.

\section{EFFECT OF HEAVY IONS INTERACTION IN MOS DEVICES}

In our irradiation laboratory test, we are interested in the effects of the interactions of high-energy heavy ions (HI) considered with enough energy to break atomic bonds and create electron-hole pairs in the material. The ionization amount is related to the total dose absorbed in the material, and is usually given in units of rads, depending upon 
the material. In the dose rate regimes of usual interest in the space environments, the main consequence of the ion energy deposition is the trapping of electrons and holes created in dielectric material, and the subsequent alteration of properties of the device.

High energy heavy ions produce charge trapping centers and defects along their path in a semiconductor material, particularly at the $\mathrm{Si}-\mathrm{SiO}_{2}$ interface of a MOS device, which can reduce the threshold voltage of the device. Also, a sharp reduction in the drain current with increasing fluence was observed, as well as, the more is the atomic weight of the ion, the more is the reduction in the drain current.

Figure 1 shows a simple view of an $n$-channel power MOSFET device. When a bias potential $\left(V_{G S}\right)$ is applied to the gate contact, an electric field appears, created across the gate oxide region and into the Si surface region immediately below the gate region. If the gate bias is sufficiently large and positive (for the $n$-channel operation), the majority carriers (holes in the $p$-body) are depleted in this surface region, and the minority carriers (electrons) are attracted to this region (for $V_{G S} \geq V_{t h}$ ). Thus, when a potential difference is applied between

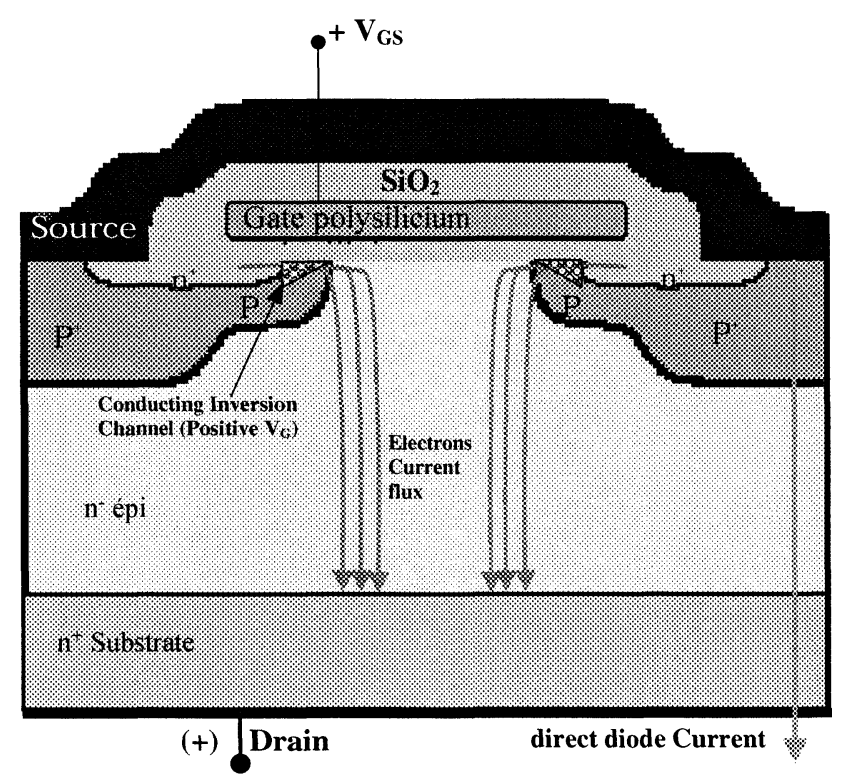

FIGURE 1 Schematic of $n$-channel VDMOSFET illustrating the normal operation. 
the drain and source contacts $\left(n^{+}-\right.$doped regions in Fig. 1), the inversion layer provides a low resistance current channel easing the electrons flow from the source to the drain. The device is then said to be turned on, and the control gate bias potential at which the channel begins to conduct appreciable current, is called the turn-on voltage or threshold voltage $\left(V_{t h}\right)$ of the device.

\subsection{Total-dose Ionization Effects}

The total dose ionization problem that occurs in this structure is due to the radiation-induced charge in the thin gate oxide region, which generates additional space-charge fields at the surface [1]. These additional induced fields result in voltage offsets or shifts in the threshold voltages of the devices, which lead to circuit degradation and failure. For example, for sufficiently large amounts of trapped positive charge, the device shown in Figure 2 may be turned on, even for zero applied gate bias. In this case, the device is said to have failed by "going depletion mode".

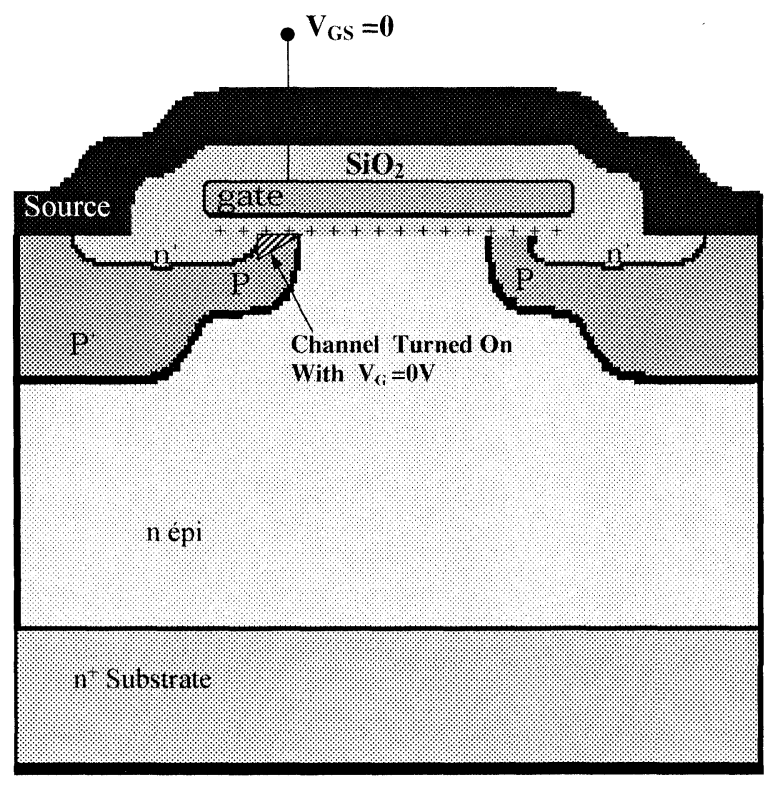

Drain

FIGURE 2 Schematic of $n$-channel VDMOSFET illustrating basic effect of total-doseionization-induced charge at the gate oxide. 
Figure 3 [2] displays a schematic energy band diagram for a MOS structure with a positive bias applied to the gate, so that free electrons in the oxide layer are swept toward the gate and holes are attracted to the $\mathrm{Si}$ substrate. The four major basic processes contributing to the radiation response of such a system are represented, which are discussed below.

As mentioned in several studies [3-5], the most sensitive part, of a MOS structure to ionizing radiations, is the oxide insulating layer $\left(\mathrm{SiO}_{2}\right)$. When the radiations pass through the oxide, the deposited energy creates electron-hole pairs. In $\mathrm{SiO}_{2}$, the radiation-generated electrons are more mobile than the holes, and are swept out of the oxide (collected at the gate electrode) in a time of the order of picoseconds [6]. However, in the first picoseconds, some fractions of the electrons and holes recombine, which depend greatly on the applied field and on the energy and type of the incident particle. The holes, which escape initial recombination, are relatively immobile and remain near their point of generation causing negative voltage shifts

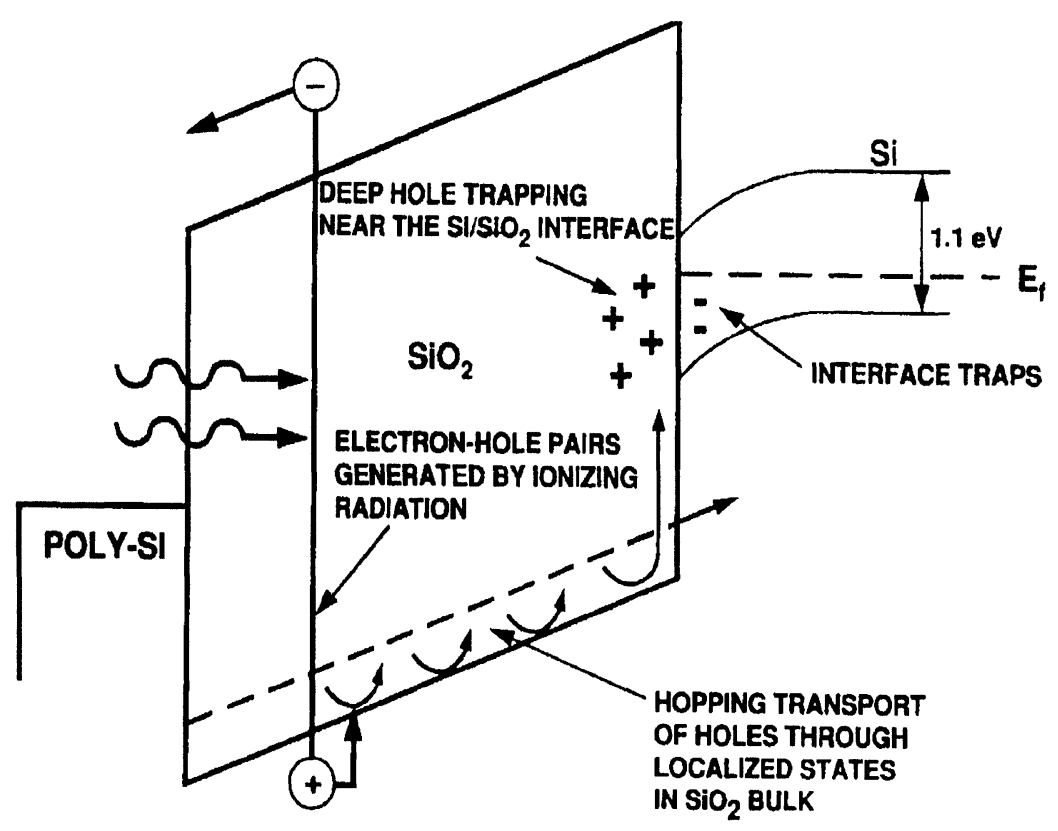

FIGURE 3 Schematic energy band diagram of $\mathrm{SiO}_{2}$ MOS structure for positive gate bias, indicating major physical processes underlying radiation response. 
in the electrical characteristics of MOS devices, e.g., in threshold voltage $\left(V_{t h}\right)$ for MOS transistors or flatband voltage $\left(V_{f b}\right)$ for MOS capacitor. These initial processes of pair creations and prompt recombinations which determine the actual charge (hole), yield in the $\mathrm{SiO}_{2}$ film and consequently the initial voltage shift constitutes the first major factor of the MOS response.

Over a period of time typically extending from $10^{-7} \mathrm{~s}$ to the order of one second (at room temperature), the holes undergo a rather anomalous stochastic hopping transport through the oxide in response to any electric fields (they move toward the $\mathrm{Si}$ substrate for the gate bias situation depicted in Fig. 3). The hole transport process, dispersive in time, is the second major factor of the MOS response. It gives rise to a short-term transient recovery in the voltage shifts and is sensitive to many variables, including a primarily applied field, temperature, and oxide thickness. When the holes reach the $\mathrm{SiO}_{2}$ interface (for positive applied gate bias), some fraction of them are captured in long-term trapping sites, causing a negative voltage shift that is not sensitive to the silicon surface potential and which can persist in time for hours to years. This long lived radiation induced voltage shift is the most commonly observed form of radiation damage in MOS device $[3,4]$. The long term trapping of holes near the $\mathrm{SiO}_{2}-\mathrm{Si}$ interface [7] constitutes the third major factor of MOS response indicated on Figure 3. Hole trapping is very sensitive to the processing of the oxide and to other variables such as field and temperature [8].

The fourth process, contributing to the MOS response, is that of a radiation induced buildup of interface traps right at the $\mathrm{SiO}_{2}-\mathrm{Si}$ interface. These are localized states with energy levels within the $\mathrm{Si}$ bandgap, they are located spatially at or near the interface, and freely exchange charge with silicon. Their occupancy is determined by the location of the Fermi level at the interface, and, consequently, the radiation induced interface traps give rise to a voltage shift component which depends on the silicon surface potential. This radiation induced threshold voltage can be theoretically expressed $[9,10]$.

\subsection{Single-event Effects}

A high-energy particle passing through a material creates displacement damage or electron-hole pairs by ionization of atoms in the crystal. 
Charge collection, as a result of the ionization interactions [11], can cause changes in the circuit operation or in the stored information, leading to what we called the Single Event Effects (SEE) [12]. An energetic ionizing particle, going through a semiconductor material, creates a track of ionization with a radius typically less than $1 \mu \mathrm{m}$ $[13,14]$. The energy deposited by an incident particle is given by its stopping power or linear transfer (LET), usually given in units of $\mathrm{MeV} \cdot \mathrm{mg}^{-1} \cdot \mathrm{cm}^{2}$.

Two kinds of SEE damages were observed in the VDMOSFETs. Which are the Single Event Burnout (SEB) and the Single Event Gate Rupture (SEGR) [15-17]. They may result in degraded performance or a catastrophic failure in these devices.

The Single-event Burnout of DMOS devices caused by a second breakdown is potentially a significant problem in the space system. The parasitic npn bipolar transistor, present in conventional DMOS structures $[18,19]$ under normal operation conditions, is turned off, but the plasma filament generated by the passage of a heavy ion is capable of locally turning it on (Fig. 4). This can lead to an avalanching in the drain depletion region and trigger the npn structure

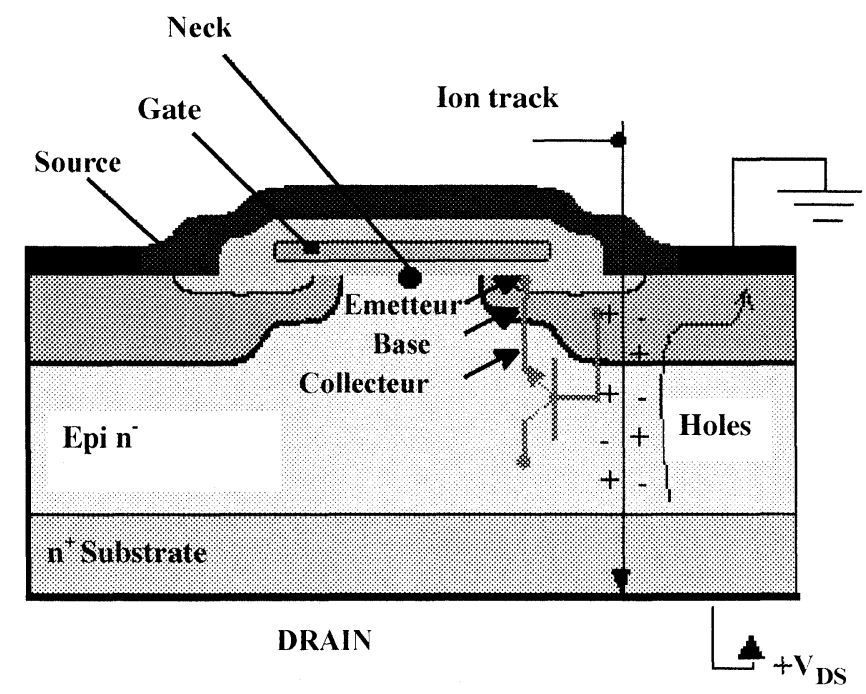

FIGURE 4 Illustration of the ion shunt effect where the high charge density along a track can turn on the bipolar device. 
into a second breakdown. This can occurs while the MOSFET is operated with a drain-source voltage exceeding the collector-emitter breakdown voltage of the parasitic bipolar device [20,21]. The plasma track initiates an avalanching in the $n$-epitaxial region, where the holes generated by the plasma column and the avalanche process travel through the resistance of the base toward the body contact giving rise to a local base potential. If this potential is sufficient to turn on the parasitic npn-transistor, then the second breakdown can be initiated. Which leads to excessive local heating and subsequent burnout of the device.

SEGR can occur when an ion strikes in the neck region of the power MOSFET. The energy deposited by the ion creates a high-density filament of electron-hole pairs in both the oxide and the silicon. Under a positive drain-source $\left(V_{D S}\right)$ bias the holes are driven toward the gate and the electrons flow toward the drain. The accumulated holes in the silicon at the $\mathrm{Si}-\mathrm{SiO}_{2}$ interface cause the electric field in the gate oxide to increase [22]. This electric field is a temporary transient field that adds to the pre-existing steady-state field in the gate oxide. If the electric field in the gate becomes sufficiently large, then oxide breakdown (i.e., SEGR) occurs [23-25].

\section{MODEL FOR JUNCTION PARAMETERS EXTRACTION}

As described in the introduction, when a positive voltage (greater than $\left.V_{t h}\right)$ is applied on the gate, and for a potential difference applied between the drain and the source, a main transistor electrons current flows horizontally from the source, through the channel region, and vertically from the drain region through the body of the device (Fig. 1). In this case, the transistor is directly biased and the bodydrain diode is reversibly biased.

In our method of the parameters junction study [26], the transistor is reverse biased and the body-drain diode is forward biased (the source is positively biased with respect to the drain), a direct current flows through the source cell across the forward biased $p-n$ junction (Fig. 1), and the conduction of the body-drain diode results in minority carriers injection into the $n$-substrate. At low gate voltage, a reverse 
transistor current can flow along the small inversion layer and the resulting current is, generally, the sum of the direct diode current and of the reverse transistor current [27]. As the channel switches OFF, for a null gate voltage, the resulting current consists in the diode current only. In our experiment, pre- and post-irradiation $I-V$ junction characteristic measurements were performed without gate bias, which allows us to understand the change due to the irradiation in the junction parameters. From the point of view of the diode direct current measurement, any tiny subthreshold channel current would appear as a current flowing through the shunt resistance (Eq. (1)).

Current $(I)$ and voltage $(V)$ values of two hundred points of experimental $I-V$ characteristics, are computer-driven via a data acquisition board and stored for modeling analysis. The description of the $I-V$ characteristics of silicon $p-n$ junctions have been thoroughly developed [28, 29]. The related implicit Eq. (1) introduces the classical parameters, series $\left(R_{s}\right)$, shunt $\left(R_{s h}\right)$ resistances, the ideality factor $(A)$, the reverse diffusion current $\left(I_{01}\right)$ and the reverse recombination current $\left(I_{02}\right)$.

$$
\begin{aligned}
I= & \frac{V-R_{s} I}{R_{s h}}+I_{01}\left[\exp \left(\frac{q}{K T}\left(V-R_{s} I\right)\right)-1\right] \\
& +I_{02}\left[\exp \left(\frac{q}{A K T}\left(V-R_{s} I\right)\right)-1\right]
\end{aligned}
$$

The electronic diffusion-recombination phenomena in the quasineutral region of the junction (reverse current $I_{01}$ ) is separated from the surface and space-charge region recombination phenomena (reverse current $I_{02}$ ). This practice is recognized through its implementation in the Spice model of the diode. A specially conceived software [30], PARADI, extracts the values of $I_{01}, I_{02}, A, R_{s}$ and $R_{s h}$ from the experimental $I-V$ diode measurements.

The evolution of the junction parameters has proved to be related to the device degradation [31].

\section{EXPERIMENTS}

Heavy ions irradiation experiments were performed at the 88 -inch Cyclotron at the University of Louvain-la-Neuve (U.C.L), Belgium. A 
lot of twenty $n$-channel VDMOSFETs (IRF130) were used for these experiments. These devices were characterized, and delidded prior to ion exposure. The open VDMOSFET structure was then irradiated with $557 \mathrm{MeV}^{132} \mathrm{Xe}^{26+}$ characterized by a LET of $63 \mathrm{MeV} \cdot \mathrm{cm}^{2} \cdot \mathrm{mg}^{-1}$, and a penetration range in the silicon exceeds $43 \mu \mathrm{m}$ : thus, this ion crosses the body-drain junction. Two kinds of experiments have been done to discuss the dose effect, then the SEE degradation processes depending upon the device operation conditions.

\subsection{Dose Effects}

In order to study the dose effect on the electrical properties of a VDMOSFET, we performed irradiation experiments with a total fluence value was about $10^{6} \mathrm{ions} / \mathrm{cm}^{2}$ equivalent to $10 \mathrm{Krad}(\mathrm{Si})$.

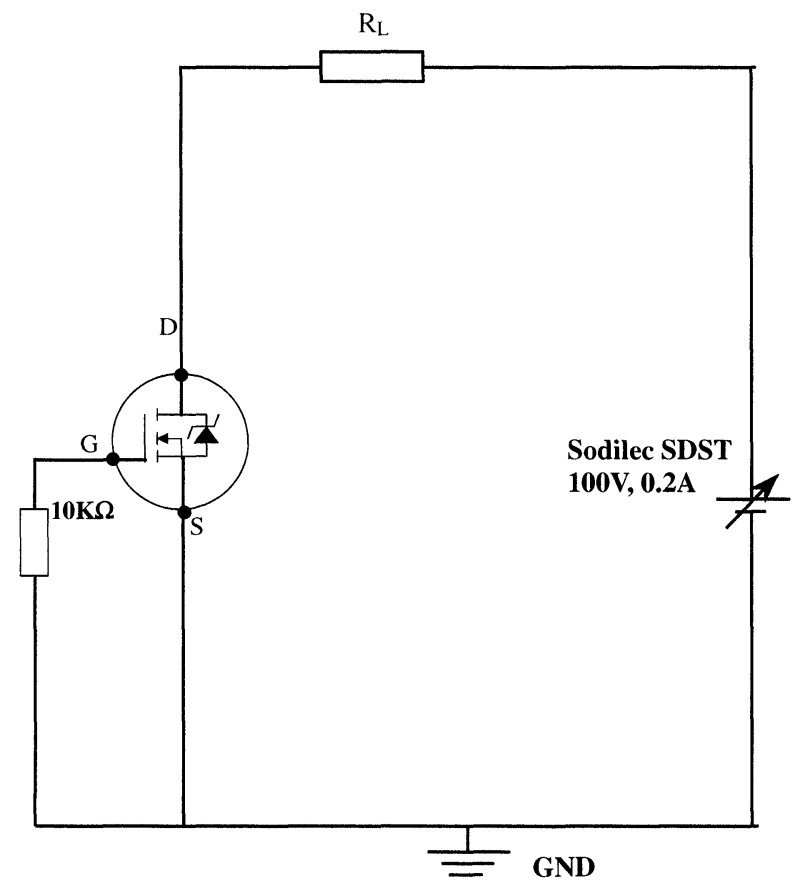

FIGURE 5 Experimental set-up used for IRF130 reverse bias under irradiation. 


\subsection{SEE Effects}

Ten VDMOSFETs of the given lot were irradiated under different conditions to study the SEE effects on the electrical properties of the body-drain junction.

During irradiation, the gate-to-source bias $V_{G S}$ was Kept at $0 \mathrm{~V}$ (Fig. 5). Drain-to-source bias under irradiation was varied from $50 \mathrm{~V}$ to $90 \mathrm{~V}$ with step values of $10 \mathrm{~V}$. For each bias value, a new component of the same lot was used. Fluence was kept fixed for all the components at $10^{5}$ ions $\cdot \mathrm{cm}^{-2}$. A resistor $R_{L}$ between the drain and the voltage regulator was used as a protective load. Experiments were performed with respectively $R_{L}=130 \Omega$ for one lot of five VDMOS FETs and $R_{L}=250 \Omega$ for another lot of five VDMOSFETs. Through this method of VDMOSFETs devices study, the role of the hot carrier degradation on the power devices can be understood.

\section{RESULTS AND DISCUSSION}

The electrical characteristics $I_{D S}-V_{D S}$ and the transfer characteristic $I_{D S}-V_{G S}$ of the VDMOSFETs (n-channel, IRF130) were determined using the setup represented in Figure 6. Figure 7 displays the dose effect on the variation of the drain current $I_{D S} v s$ drain to source

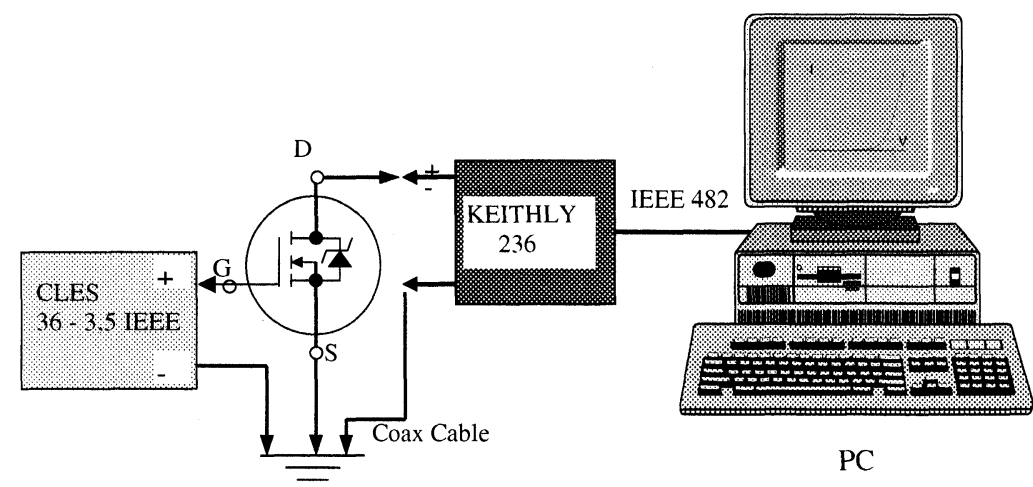

FIGURE 6 Experimental setup for device characterization. 


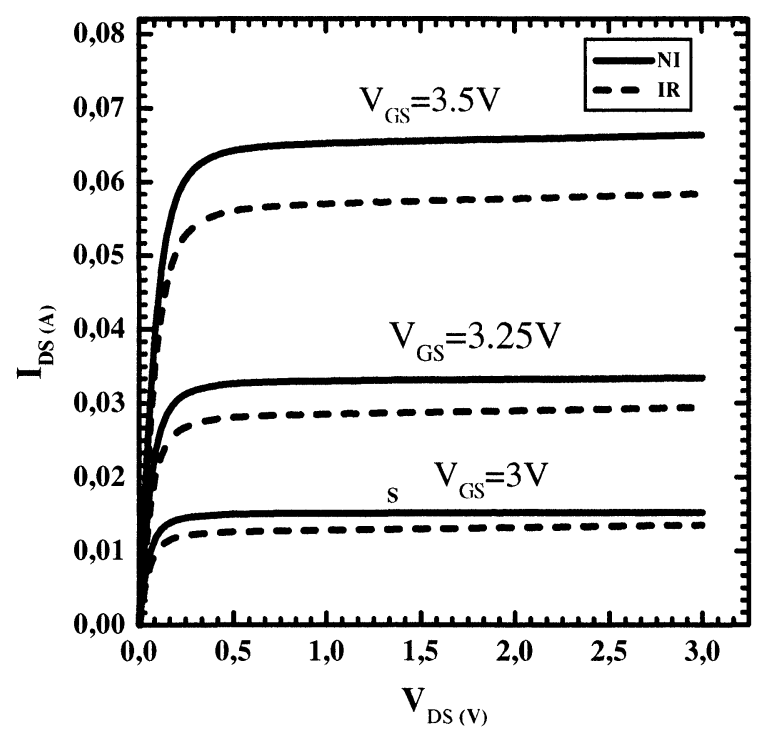

FIGURE 7 Pre- and post-irradiation electrical characteristic $I_{D S}=f\left(V_{D S}\right)$.

voltage $V_{D S}$ at three fixed gate voltages $V_{G S}(3,3.25,3.5$ Volt $)$ for the irradiated devices. These curves confirm an expected increase of current $I_{D S}$ as $V_{G S}$ increases for both non irradiated and irradiated devices. Higher $V_{G S}$ values yield larger $n$-channel and consequently larger $I_{D S}$ values. On the other hand, Figure 7 displays a difference, between the $I_{D S}$ values of the same device before and after irradiation, increasing when $V_{G S}$ increases. The irradiation creates trapping centers at the $\mathrm{Si}-\mathrm{SiO}_{2}$ interface and in a few nanometers inside the silicon near the $\mathrm{Si}-\mathrm{SiO}_{2}$ interface. When these centers are situated in the $n$-channel, they trap the channel electrons which diminishes the $I_{D S}$ values. Moreover, as the $n$-channel width increases, more traps are included in the channel which enhances the relative $I_{D S}$ decrease which appears (Fig. 7) when $V_{G S}$ increases.

Figure 8 shows the transfer characteristic after the $10 \mathrm{Krad}(\mathrm{Si})$ irradiation dose by the heavy ions. The threshold voltage $V_{t h}$ was determined in the saturation region where the current of the VDMOSFET is given [32] by:

$$
I_{D S(\mathrm{sat})} \approx \mu_{n} C_{o x} W / L\left(V_{G S}-V_{t h}\right)^{2}
$$




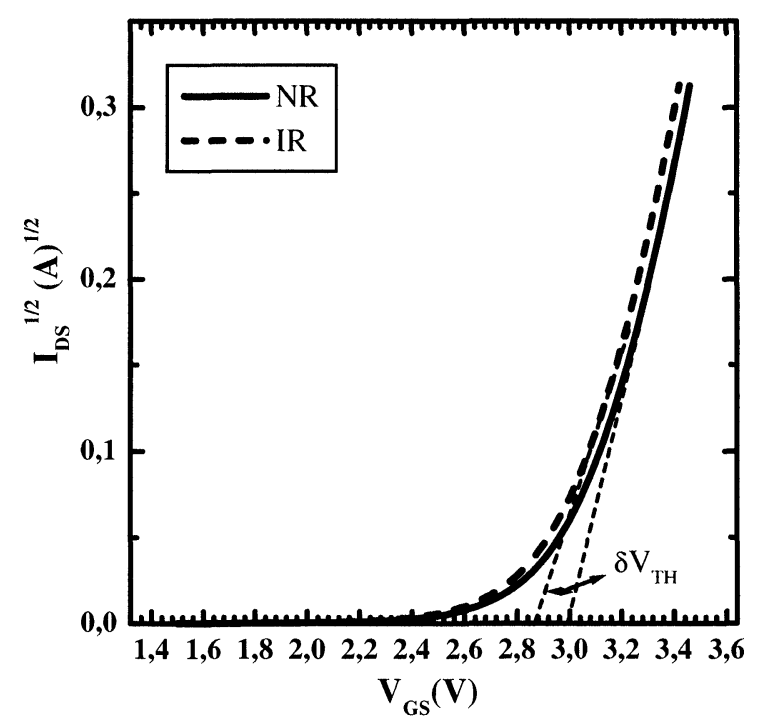

FIGURE 8 Pre- and post-irradiation electrical characteristic $I_{D S}=f\left(V_{G S}\right)$.

By plotting $\sqrt{I_{D S}}$ versus $V_{G S}$ (for a given $V_{D S}>V_{D S(\text { sat) }}$ ), the threshold voltage can be deduced from the linearly extrapolated value at the $V_{G S}$ axis. This result shows a decrease of $V_{t h}$. This observed decrease is due to accumulated positive charge due to the heavy ion irradiation in the $\mathrm{SiO}_{2}$ layer. The accumulated charge creates an electrical field that modifies the oxide silicon interface potential leading to $V_{t h}$ variations.

A description of the physical degradation processes may be done by considering the evolution of the body-drain junction properties with the irradiation conditions. For each of the ten MOSFETs mentioned in part 4.2 , body drain junction parameters were extracted with the method exposed in part 3, before and after irradiation. Figure 9 shows an increase in the reverse recombination current $I_{02}$. It also points out that the $I_{02}$ shift is more important for high $V_{D S}$ values. These results can be explained by the effects induced in the device as a consequence of the heavy ion interactions during irradiation. The electric field due to the applied positive bias (to the drain) causes the holes and electrons, generated in the silicon by the ion interaction, to move respectively toward the $\mathrm{Si}-\mathrm{SiO}_{2}$ interface (at the channel region) and toward the drain contact. This enhances the carrier flux 


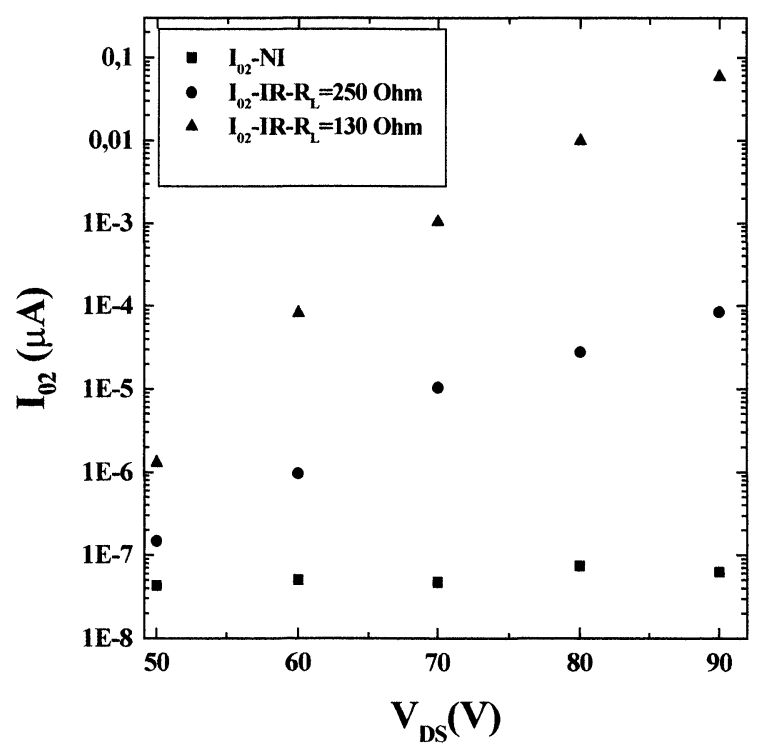

FIGURE 9 The body-drain junction reverse recombination current $v s$ the reverse voltage applied under irradiation.

[33]. The location of the maximum electric field moves from the body-drain junction to the $n-n^{+}$boundary in the drain region. The electric field can then reach a high level inducing an avalanche effect [34] that yields high energy majority carriers injection toward the $\mathrm{Si} / \mathrm{SiO}_{2}$ interface [35]. This effect is more important for higher values of $V_{D S}$. A part of these hot holes attains enough energy to cross the potential barrier at the $\mathrm{Si}-\mathrm{SiO}_{2}$ interface $[36,37]$ and creates fast surface states at the $\mathrm{Si}-\mathrm{SiO}_{2}$ interface producing a change in the surface potential [38-40].

The main result shown by Figure 9 concerns the lower $I_{02}$ shift obtained for higher $R_{L}$ value. Since $R_{L}$ limits the current in the external circuits, the number of hot carriers is limited which reduces the previous degradation process.

Figure 10 displays the evolution of the shunt resistor $\left(R_{s h}\right)$ during the irradiation process. The decrease of $R_{s h}$ after irradiation is a signature of junction properties degradation. The degradation is more important for high values of the drain bias. We also observed that the degradation is lower for great values of the resistor $R_{L}$. This evolution $v s V_{D S}$ leads to relate the irradiation effect to the creation of fast surface states by hot carriers. 


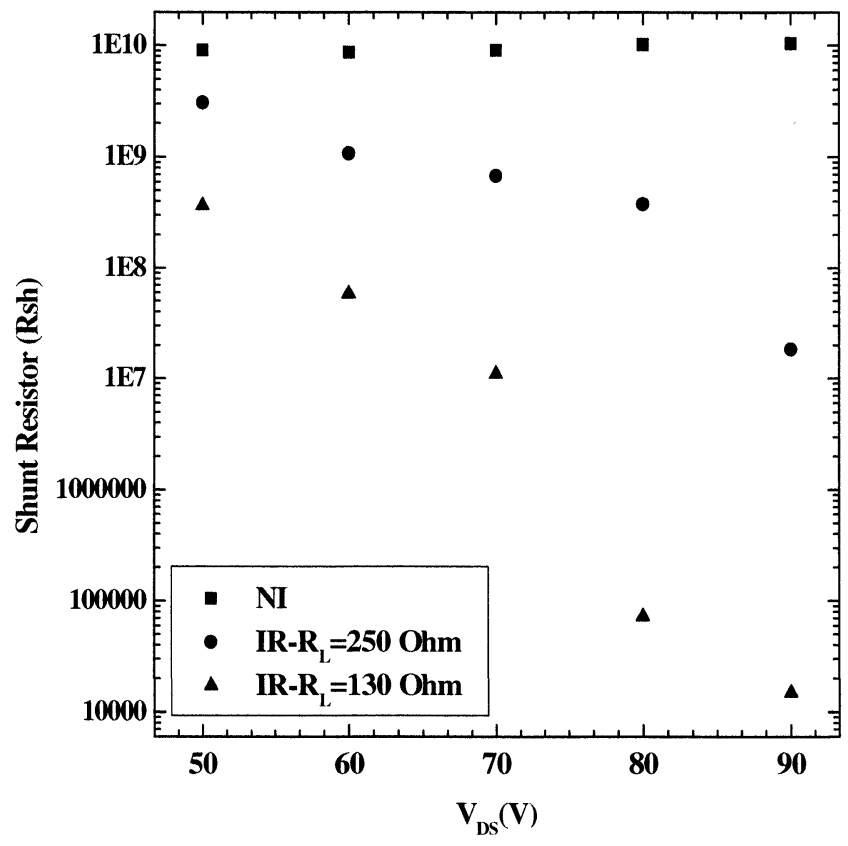

FIGURE 10 The body-drain junction shunt resistance, $R_{s h}$, vs the reverse voltage applied under irradiation.

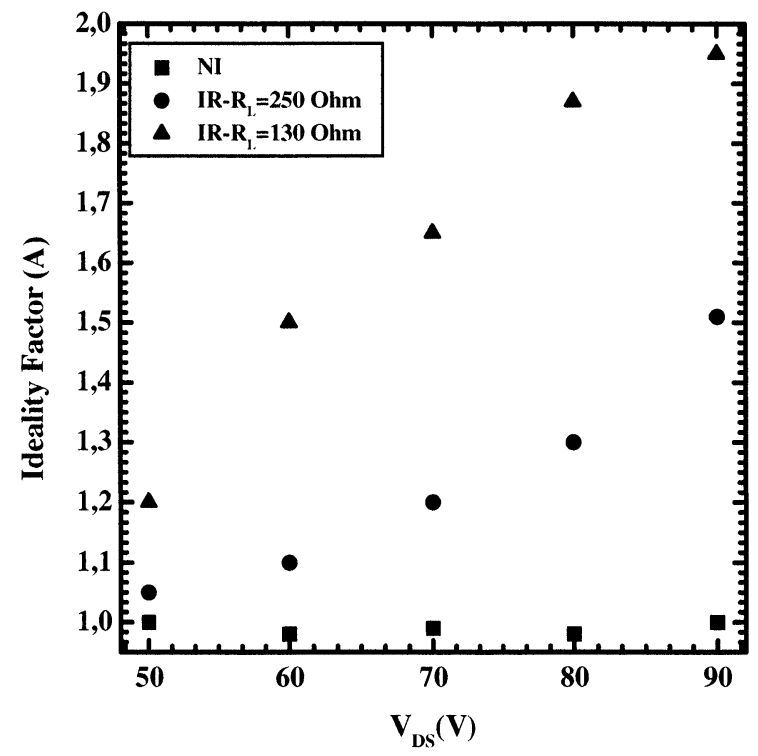

FIGURE 11 The body-drain junction ideality factor, $A$, vs the reverse voltage applied under irradiation. 
The ideality factor $(A)$ values of the body-drain junction shows a large sensitivity (Fig. 11) to the applied drain voltage during irradiation. Values higher than unity [41] indicate an increase of the surface and space charge recombination current. It is related to the increase of the traps density and denotes a degradation of the junction. This result is correlated with the high sensitivity of the reverse recombination current to the hot carriers degradation processes due to heavy ion interaction in the devices. Resistor $R_{L}$ appears as a protective load since high $R_{L}$ values lower the radiation induced degradation.

\section{CONCLUSION}

Basic physical processes which determine the radiation response of electronic devices have been discussed. Total dose effects involve the creation and the trapping of charges in dielectrics and the creation of interface traps. Single event effects are associated with the charge generated by the passage of a single high energy particle through a device structure. The subsequent charge multiplication and the drain avalanche hot hole injection result in a degradation of the device properties.

The degradation of the physical parameters of the body-drain junction of heavy ions irradiated power VDMOSFET, has been studied by an innovative and powerful method for microelectronics device characterization. Increase in reverse current recombination has been found related to the drain applied voltage and the resulting hot-holes injection of drain avalanche. An increase of the shunt resistance is also pointed out and depends on the hot-holes injection effect. The ideality factor degradation is found to be related to the same effect. The magnitude of the device degradation has been shown to be weakened by the introduction of a drain resistor load.

\section{Acknowledgements}

HI irradiation was performed at the U.C.L. (University of Louvain-laNeuve, B) Cyclotron with support of the European T.M.R. program. The authors wish to thank Guy Berger for his help and technical assistance. 


\section{References}

[1] Ristic, G. S., Jaksic, A. B. and Pejovic, M. M., "Latent interface-trap buildup in power MOSFETs: new experimental evidence and numerical simulation", RADECS 99, 13-17 September, 1999, Fontervraud France, p. H14.

[2] Mclean, F. B., Boesch, H. E. and Oldham, T. R. (1989). "Electron-Hole Generation, Transport, and Trapping in $\mathrm{SiO}_{2}$ ", In: Ionizing Radiation Effects on MOS Devices and Circuits, edited by Ma, T. P. and Dressendorfer, P. V., John Wiley \& Sons, New York, p. 87.

[3] Buchanan, D. A. and DiMaria, D. J. (1990). "Interface and Bulk trap Generation in MOS Capacitors", J. Appl. Phys., 67.

[4] Fleetwood, D. M. (1992). "Border Traps in MOS Devices", IEEE Trans, Nucl. Sci., 39, 296.

[5] Boesch, H. E. Jr. (1982). "Interface-State Generation in Thick $\mathrm{SiO}_{2}$ Layers", IEEE Trans. Nucl. Sci., 29, 1446.

[6] van Lint, V. A. J., Flanagan, T. M., Leadon, R. E., Naber, J. A. and Rogers, V. C. (1980). "Mechanisms of Radiation Effects in Electronic Materials", Vol. 1, WileyInterscience, New York.

[7] Saigné, F. et al. (1997). "Experimental validation of an accelerated method of oxide-trap-level characterization for predicting long term thermal effects in MOS Devices", IEEE Trans. Nucl. Sci., 44, 2006.

[8] Dusseau, L. et al. (1997). "Prediction of low-rate effects in power metal oxide semiconductor field effect transistors based on isochronal annealing measurements", J. Appl. Phys., 81, 2437-2441.

[9] Shanfield, Z. and Moriwaki, M. M. (1985). "Radiation Induced Hole Trapping and Interface State Characteristics of Al-gate and poly-Si Gate MOS Capacitors", IEEE Trans. Nucl. Sci., 32, 3939.

[10] Boesch, H. E. Jr., McLean, F. B., Benedetto, J. M. and McGarrity, J. M. (1986). "Saturation of Threshold Voltage Shift in MOSFET's at High Total Dose", IEEE Trans. Nucl. Sci., 33, 1191.

[11] Dodd, P. E. (1997). "Charge Collection and SEU from Angled Ion Strikes", IEEE Trans. Nucl. Sci., 44, 2256.

[12] Koga, R. (1996). "Single-event Effect Ground Test Issues", IEEE Trans. Nucl. Sci., 43, 670 .

[13] Howard, J. W. et al. (1994). "A novel approach for measuring the radial distribution of charge in a heavy-ion track", IEEE Trans. Nucl. Sci., 41, 2077.

[14] Dodd, P. E. (1996). "Device simulation of charge collection and SEU", IEEE Trans. Nucl. Sci., 43, $561-575$.

[15] Nichols, D. K. et al. (1994). "Observations of Single Event Failure in Power MOSFETs", IEEE NSREC Data Workshop Record, pp. 41-54.

[16] Johnson, G. H. (1993). "Simulating Single-event Burnout of $n$-Channel Power MOSFETs", IEEE Trans. Elect. Dev., 40, 1001-1008.

[17] Johnson, A. H., Palau, J. M., Dachs, C., Galloway, K. F. and Schrimpf, R. D. (1996). "A Review of the Techniques Used for Modeling Single-event Effects in Power MOSFETs", IEEE Trans. Nucl. Sci., 43, 546.

[18] Wrobel, T. F., Coppage, F. N., Hash, G. L. and Smith, A. (1985). "Current Induced Avalanche in Epitaxial Structures", IEEE Trans. Nucl. Sci., 32, 3991.

[19] Hohl, J. H. and Johnson, G. H. (1989). "Features of the Triggering Mechanism for Single Event Burnout of Power MOSFETs", IEEE Trans. Nucl. Sci., 36, 2260.

[20] Hohl, J. H. and Galloway, K. F. (1987). "Analytical Model for Single-event Burnout of Power MOSFETs", IEEE Trans. Nucl. Sci., 34, 1275.

[21] Liu, J., Schrimpf, R. D., Massengill, L., Galloway, K. F. and Attia, J., "SEB Circuit-Level in $n$-Channel Power MOSFETs", RADECS 99, 13-17 September, 1999, Fontervraud France, p. F-8. 
[22] Scarpa, A., Paccagnelle, A., Montera, F. and Fuochi, P. G. (1997). "Ionization Radiation Induced Leakage Current on Ultra-Thin Gate Oxide", IEEE Trans. Nucl. Sci., 44, 1818.

[23] Brews, J. R., Allenspach, M., Schrimpf, R. D., Galloway, K. F., Titus, J. L. and Weathley, C. F. (1993). "A Conceptual Model of Single-event Gate Rupture in Power MOSFETs", IEEE Trans. Nucl. Sci., 40, 1959.

[24] Allenspach, M., Mouret, I., Titus, J. L., Weathley, C. F. Jr., Pease, R. L., Brews, J. R., Schrimpf, R. D. and Galloway, K. F. (1995). "Single-event Gate Rupture in Power MOSFETs; Prediction of Breakdown Biases and Evaluation of Oxide Thickness Dependence", IEEE Trans. Nucl. Sci., 42, 1922.

[25] Allenspach, M., Brews, J. R., Mouret, I., Schrimpf, R. D. and Galloway, K. F. (1994). "Evaluation of SEGR Threshold in Power MOSFETs", IEEE Trans. Nucl. Sci., 41, 2160.

[26] De la Bardonnie, M., Mialhe, P., Bandada, E., Blompain, E., Hoffmann, A. and Charles, J.-P. (1998). "Diode Parameter Determination Applied to LDDMOSFETs for Device Characterization", Active and Passive Elec. Comp., 20, $157-163$.

[27] L., Le Bras. Matti Bendada, Mialhe, P., Blompain, E. and Charles, J.-P. (1994). "Recombination Via Radiation-Induced Defects in Field-Effect Transistor", J. Appl. Phys., 76, 5676.

[28] Sah, C. T., Noyce, R. N. and Shockley, W. (1957). "Carrier Generation and Recombination in $p-n$ Junctions and $p$ - $n$ Junction Characteristics", Proc. IRE45, p. 1228.

[29] Wolf, M. and Rauschenbach, H. (1963). "Series Resistance Effects on Solar Cell Measurements", Adv. Energy Convers., 3, 455.

[30] Charles, J.-P., Mekkaoui-Alaoui, I., Bordure, G. and Mialhe, P. (1985). "A Critical Study of the Effectiveness of the Single and Double Exponential Models." SolidState Electron., 28, 807.

[31] de la Bardonnie, M., Toufik, N., Salame, C., Dib, S., Mialhe, P., Hoffmann, A. and Charles, J.-P. (1999). "Junction parameters for silicon devices characterization", Microelectron. Reliab., 39, 751.

[32] Sze, S. M., Physics of Semiconductor Devices, Ch. 8, John Wiley, New York, 1981.

[33] Wrobel, T. F., Coppage, F. N., Hash, G. L. and Smith, A. J. (1992). "Current Induced Avalanche in Epitaxial Structure", IEEE Trans. Nucl. Sci., 39, 1636.

[34] de la Bardonnie, M., Jiang, D., Kerns, S. E., Kerns, D. V. Jr., Mialhe, P., Charles, J.-P. and Hoffmann, A. (1999). "On the Aging of Avalanche Light Emission from Silicon Junction", IEEE Trans. Elec. Dev., 46, 1243.

[35] Rafi, J. M. and Campabadal, F. (1999). "Hot-hole-induced interface states buildup on deep-submicrometer LDD nMOSFETs", Microelectronique Reliability, 39, 869.

[36] Gesh, H., Leburton, J.-P. and Dorda, G. (1982). "Generation of Interface States by Hot Hole Injection in MOSFETs", IEEE Trans. Nucl. Sci., 29, 913.

[37] Hera, H., Okamoto, Y., and Ohnuma, H. (1970). "Anew instability in MOS Transistor caused by Hot Hole injection from drain Avalanche Plasma into Gate Oxide", Japan, J. Appl. Phys., 9, 1103.

[38] Dreesen, R., Croes, K., Manca, J., De Ceuninck, W., De Schepper, L., Pergoot, A. and Groeseneken, G. (1999). "Modelling hot-carrier degradation of LDD NMOSFETs by using a high-resolution measurement technique", Microelectron. Reliab., 39, 785.

[39] Jahan, C., Bruyère, S., Ghibaudo, G., Vincent, E. and Barla, K. (1999). "Model for the Oxide Thickness Dependence of SILC Generation Based on Anode Hole Injection Process", Microelectron. Reliab., 39, 791.

[40] Sung H., Hong, Sang M., Nam, Byung O., Yun, Byung J., Lee, Chong G., Yu and Jong T., Park (1999). "Temperature Dependence of Hot Carrier Induced MOSFET Degradation at Low Gate Bias", Microelectron. Reliab., 39, 809.

[41] Sah, C. T. (1986). "High Efficiency Crystalline Silicon Solar Cells", Solar Cells, 17, 1. 

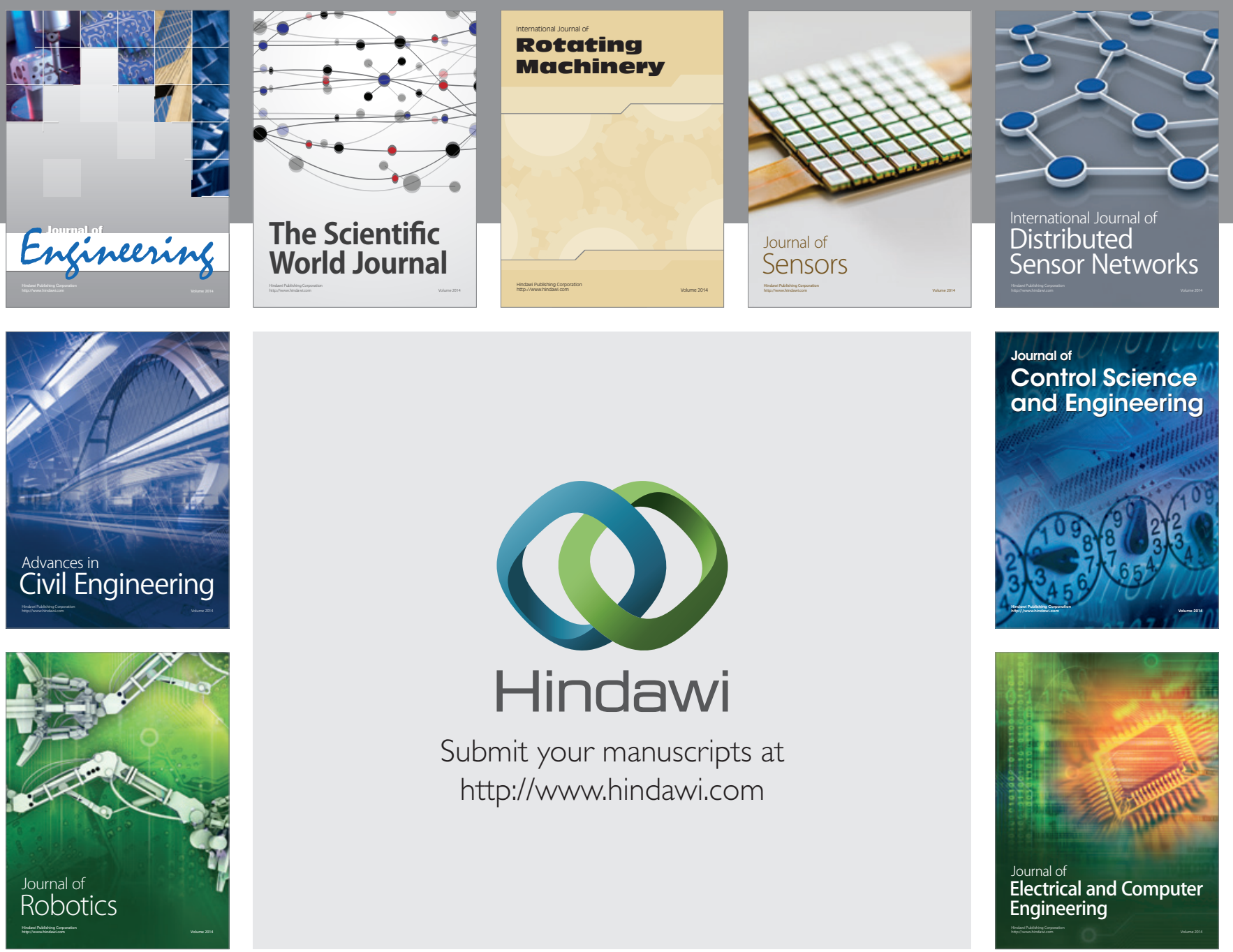

Submit your manuscripts at

http://www.hindawi.com
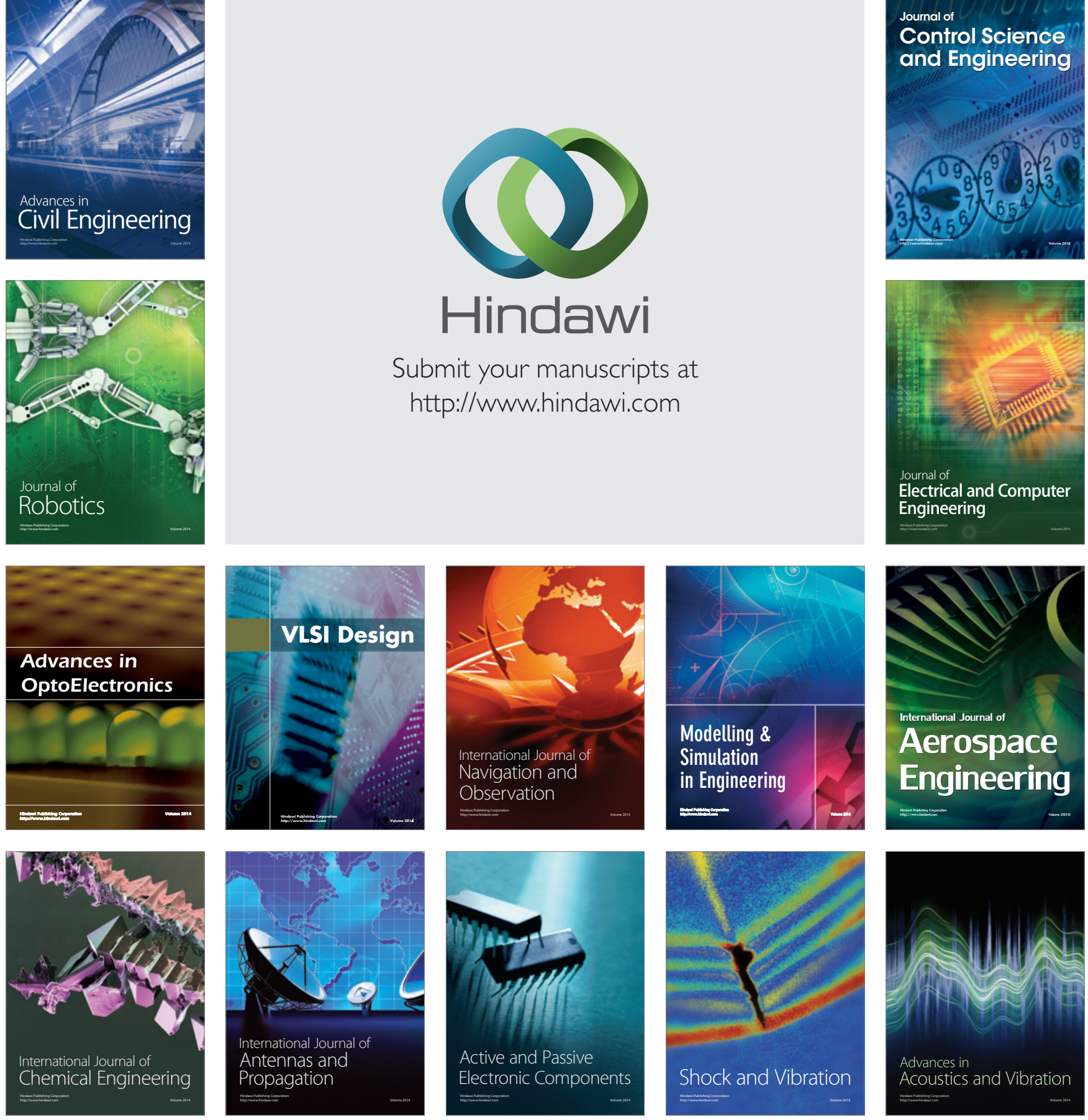